\title{
Tumor desmoide intraabdominal. Presentación de caso clínico y revisión de la literatura
}

\author{
Intraabdominal desmoid tumor. Clinical clinical case and literature review
}

Luis J. Palacios-Fuenmayor ${ }^{*}$, Ana M. Naranjo-Isaza², Ossian Fuentes ${ }^{3}$, María F. Palacio ${ }^{4}$, Alfredo Martelo ${ }^{5}$, Luis R. Gómez y Héctor García ${ }^{6}$

${ }^{1}$ Servicio de Cirugía Oncólogica, Instituto de Cancerología Las Américas, AUNA, Medellín; ${ }^{2}$ Servicio de Cirugía General, Universidad CES, Medellín; ${ }^{3}$ Servicio de Cirugía General, Universidad Militar Nueva Granada, Bogotá; ${ }^{4}$ Servicio de Cirugía General, Instituto de Cancerología Las Américas, AUNA, Medellín; ${ }^{5}$ Servicio de Patología Las Américas, AUNA, Medellín; ${ }^{6}$ Servicio de Oncología Clínica, Instituto de Cancerología Las Américas, AUNA Medellín; ${ }^{7}$ Servicio de Epidemiología, Instituto de Cancerología Las Américas, AUNA, Medellín. Colombia

\section{Resumen}

Los tumores desmoides son afecciones benignas raramente reconocidas de manera inicial por su baja incidencia. Se caracterizan por un comportamiento local agresivo y altas tasas de recurrencia. Su presentación puede ser extraabdominal o intraabdominal. Presentamos el caso de una paciente de 15 años con cuadro de dolor abdominal de 3 meses de evolución, en la que se documentó una gran masa intraabdominal con diagnóstico histológico de tumor desmoide y fue sometida a múltiples intervenciones quirúrgicas con las que se logró una resección $R 1$ (microscópicamente positivo). Se descartó su asociación con embarazo, trauma abdominal, cirugías previas y síndromes genéticos.

Palabras Clave: Tumor desmoide. Intraabdominal. Cirugía.

\begin{abstract}
Desmoid tumors are clinical entities rarely diagnosed at an initial presentation because of its low incidence, they are characterized by a locally aggressive presentation and high rates of local relapse. Its presentation can be intra- or extra-abdominal. We present a clinical case of a female, 15 year old patient, with three months of abdominal pain, a giant intra-abdominal mass was diagnosed with histologic diagnosis of desmoid tumor. Several surgical procedures were performed, having a las a $R 1$ resection (focally microscopic margins). In this case association with pregnancy, abdominal trauma, previous surgeries and genetic syndromes were discarded.
\end{abstract}

Key Words: Desmoid tumor. Intra-abdominal. Surgery.

\section{Introducción}

Los tumores desmoides son neoplasias benignas caracterizadas por una proliferación de células miofibroblásticas. Poseen bajo potencial metastásico, pero una alta capacidad de invasión local y un comportamiento recurrente de forma agresiva. Representan una mínima fracción de todos los tumores de

\section{Correspondencia:}

*Luis J. Palacios-Fuenmayor

Diagonal, 75b, 2A-80/140 
tejidos blandos en general y su presentación más común es en el abdomen. En ocasiones se encuentran asociados al síndrome de poliposis adenomatosa familiar o síndrome de Gardner.

A continuación presentamos el caso de una paciente de 15 años, previamente sana, con un cuadro de dolor abdominal de 3 meses de evolución, en la que se documentó un tumor desmoide intraabdominal de aparición esporádica y fue sometida a intervenciones quirúrgicas lográndose una resección R1 (microscópicamente positivo).

\section{Presentación del caso}

Mujer de 15 años, de raza mestiza, ocupación estudiante, sin antecedentes médicos, quirúrgicos ni familiares. Consulta en agosto de 2016 por un cuadro clínico de 3 meses de evolución de dolor en epigastrio, no irradiado, sin otra sintomatología asociada. De manera inicial, por medicina general se le realiza estudio de endoscopia digestiva alta, en el que se describe «Importante compresión extrínseca del cuerpo gástrico». Se realizó resonancia magnética contrastada de abdomen y se visualizó una lesión intraabdominal sólida neoplásica que sugiere origen mesenquimal (Fig. 1). Posteriormente, la biopsia con aguja gruesa de dicha lesión guiada por ecografía confirmó el tumor desmoide.

En junio de 2016 la paciente es sometida a resección de tumor intraabdominal, hemicolectomía derecha ampliada y anastomosis íleo-descendente. En su tercer día de posoperatorio, debido a taquicardia y fiebre persistente asociadas a elevación de los reactantes de fase aguda y hallazgos en la tomografía de abdomen contrastada consistentes con neumoperitoneo y líquido libre intraabdominal sin extravasación de medio de contraste, se decide intervenir quirúrgicamente de nuevo. Se confirma una anastomosis indemne, sin necrosis y sin áreas macroscópicas de fugas. Se realiza cierre diferido de la herida quirúrgica dada la presencia de signos clínicos de infección. Al día 17 de posoperatorio se da egreso a la paciente. El reporte definitivo de patología indica fibromatosis tipo tumor desmoide de $30 \mathrm{~cm}$ en su diámetro mayor, hemicolectomía derecha con margen de resección libre pT2bpNo (Fig. 2). Sin recibir tratamientos adicionales, debido al reporte de márgenes patológicos negativos, cirugía R0.

La paciente continúa seguimiento posoperatorio trimestral con estudio de tomografía abdominal, sin signos de recurrencia local y colonoscopia negativa para

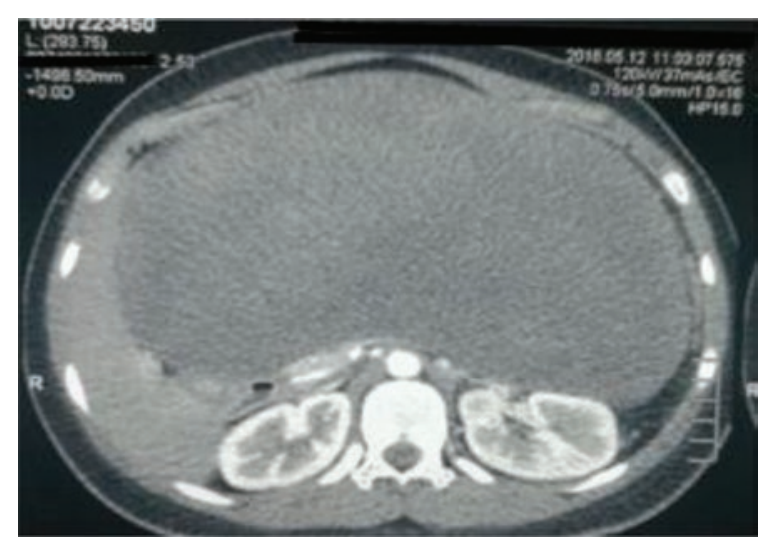

Figura 1. Tomografía de abdomen contrastada (junio de 2016) que muestra una lesión tumoral de densidad heterogénea con localización intraabdominal (cortesía de Luis J. Palacios).

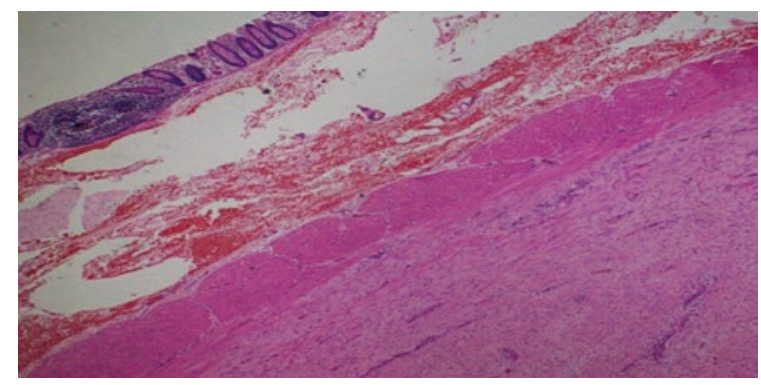

Figura 2. Representación de la pared colónica (superior derecha), en íntimo contacto con el tumor (inferior izquierdo). H\&E x 40 (cortesía de Alfredo Martelo).

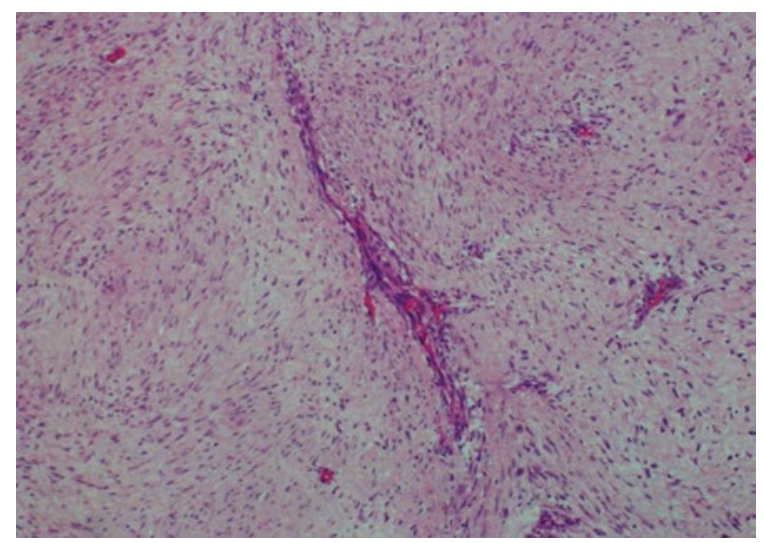

Figura 3. Tumor con estroma colagenizado con vasos sanguíneos pequeños y proliferación de células fusiformes. H\&E x 100 (cortesía de Alfredo Martelo).

pólipos intestinales. En marzo de 2018 refiere sensación de masa no dolorosa en el epigastrio, por lo que se realiza tomografía abdominal contrastada y se observa una lesión con densidad de tejido blando de aproximadamente $21 \times 50 \mathrm{~mm}$ localizada hacia la región del epigastrio, adherida a la pared abdominal anterior y sin un plano de clivaje de separación de esta. Se realiza biopsia con aguja gruesa y los resultados son compatibles con tumor desmoide (Figs. 3 a 5). En 


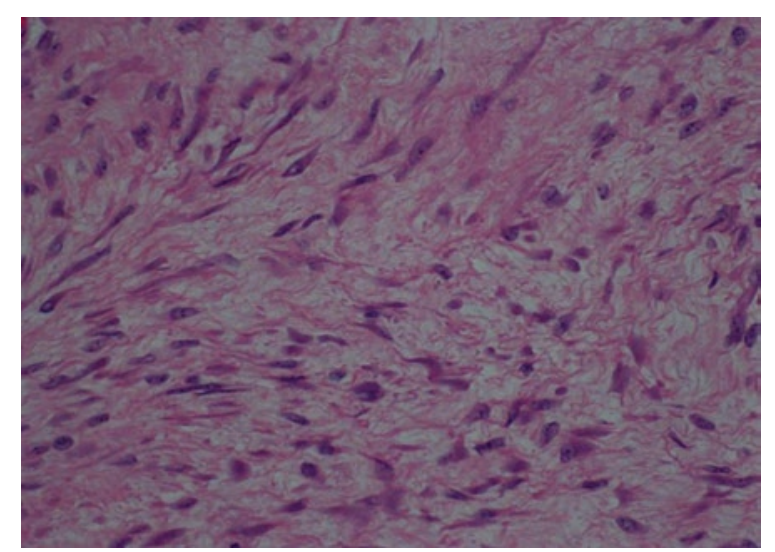

Figura 4. Detalle de las células tumorales, con características de miofibroblastos. H\&E × 400 (cortesía de Alfredo Martelo).

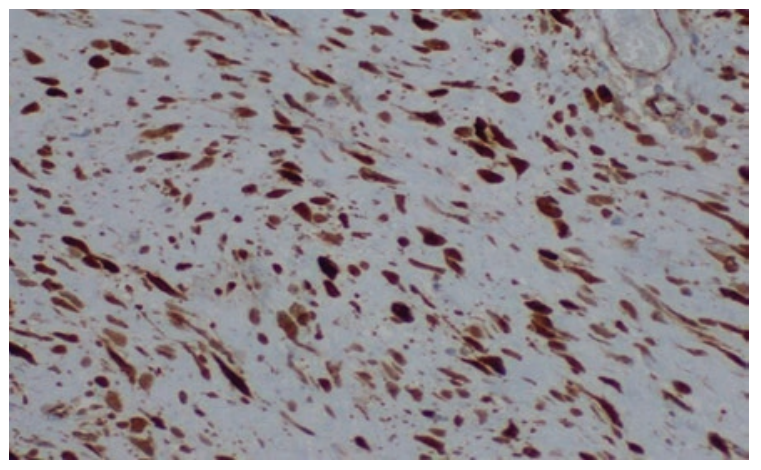

Figura 5. Inmunohistoquímica. El marcador $\beta$-catenina muestra positividad nuclear fuerte y difusa en las células tumorales. Coloración de inmunohistoquímica (marcador $\beta$-catenina) × 400 (cortesía de Alfredo Martelo).

mayo del mismo año se realizan resección del tumor de pared abdominal y eventrorrafia, identificándose un tumor desmoide en la pared abdominal, en la línea media supraumbilical subxifoidea, de $10 \times 10 \mathrm{~cm}$, con compromiso de la fascia anterior de los rectos abdominales y de la piel, y hacia la profundidad compromiso del lóbulo hepático izquierdo, segmento 2, extremo distal. Se obtiene reporte definitivo de patología de la lesión con ampliación de márgenes, que indica lesión compatible con recidiva de tumor desmoide, tamaño $9 \mathrm{~cm}$, con hallazgos en ampliación de margen distal lateral derecho en contacto con el tumor en un trayecto de $2 \mathrm{~mm}$.

Siendo el caso de una paciente joven, sometida en el último procedimiento a una reconstrucción compleja de la pared abdominal (Fig. 6), con una enfermedad de progresión lenta, se realiza junta de especialistas institucional en la que se decide en el momento no someter a la paciente a intervenciones quirúrgicas ni tratamientos médicos adicionales, basados en las guías de tratamiento institucional, donde las resecciones R1 también son aceptables para dar un manejo

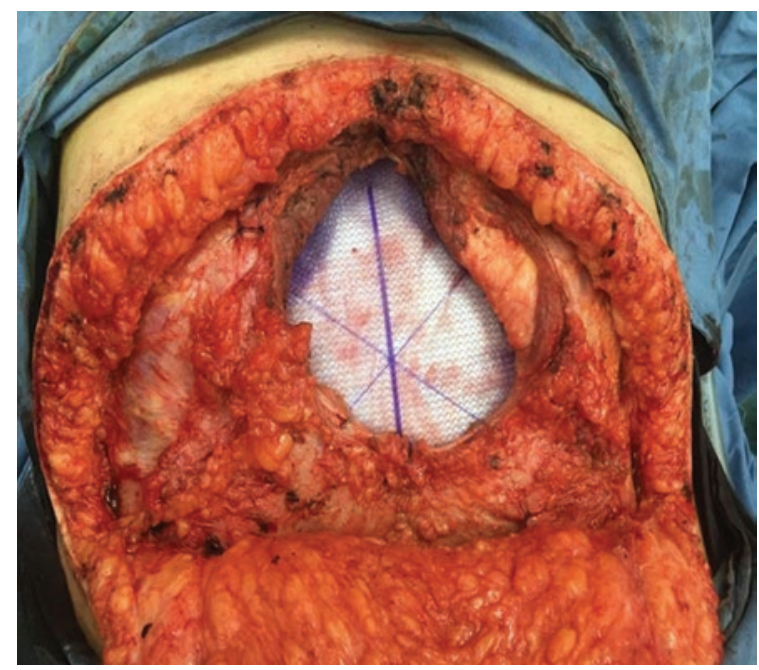

Figura 6. Imagen transoperatoria (abril de 2018). Defecto supraumbilical sin lograr el cierre en la línea media, que requiere colocación de malla separadora de componentes de $30 \times 30 \mathrm{~cm}$ (cortesía de Luis J. Palacios).

adyuvante, aunque pendiente de estudios de mayor validación.

\section{Discusión}

Los tumores desmoides o fibromatosis tipo desmoide son una afección rara y benigna caracterizada por una proliferación fibroblástica/miofibroblástica que puede aparecer en cualquier localización del cuerpo. La ausencia de cápsula y la presencia de infiltración de los tejidos adyacentes de manera multifocal generan una alta tasa de recurrencia local, lo que hace de su tratamiento un verdadero reto médico'.

Presentan una incidencia de 2-4 casos por millón al año, representando el $0.03 \%$ de todas las neoplasias y menos del $3 \%$ de los tumores de los tejidos blandos. Con mayor frecuencia aparecen en las mujeres, con una relación 2:1 respecto al hombre, y en el grupo de edad de 25-35 años ${ }^{1}$.

La mayoría de los casos (80\%) se presentan de manera esporádica, pero un $2-15 \%$ de los pacientes con tumor desmoide tienen asociada poliposis adenomatosa familiar o síndrome de Gardner; la localización anatómica típica de la lesión en estos pacientes es el mesenterio del intestino delgado ${ }^{2-4}$. Se ha evidenciado que el gen APC (Adenomatous polyposis coli) participa en el control de los niveles de $\beta$-catenina en el citoplasma. La mutación del APC presente en la poliposis adenomatosa familiar no permite la degradación de la $\beta$-catenina, generando la translocación de esta al núcleo y afectando la transcripción génica. La $\beta$-catenina es una proteína codificada por 
el gen CTNNB1 que regula la adhesión celular en el citoplasma, así como la transcripción de genes en el núcleo. Forma parte del complejo proteínico cadherina y actúa a través de la vía de señalización intracelular Wnt. Las mutaciones del APC no se encuentran presentes en la mayoría de los casos esporádicos, pero se describen mutaciones de CTNNB1 en el 85$88 \%$ de estos ${ }^{5,6}$.

La etiología del tumor desmoide no se encuentra plenamente establecida. Puede aparecer en cicatrices de cirugías previas, principalmente por procedimientos abdominales; en especial, en pacientes con poliposis adenomatosa familiar. El sitio de aparición más común en los casos esporádicos sigue siendo la pared abdominal ${ }^{7}$. Se describe un mayor riesgo de tumor desmoide posterior al embarazo, pero no se ha logrado demostrar una relación directa entre los niveles de estrógenos y el tamaño de la lesión. La expresión de receptores de esteroides sexuales por el tumor es inconstante; de acuerdo con esto, y ante la ausencia de ensayos clínicos aleatorizados, la terapia hormonal aislada no se ha estandarizado en esta enfermedad ${ }^{8}$.

La aparición de un tumor desmoide en los sitios de cicatrices quirúrgicas refleja una alteración en el proceso de reparación celular, y hace evidente la implicación de la $\beta$-catenina en este fenómeno, la cual se eleva de manera transitoria en el fibroblasto durante los procesos de cicatrización. A pesar de que las células del tumor desmoide son similares a fibroblastos con abundante matriz de colágeno, no se ha descrito como su célula predecesora. Se han identificado marcadores de superficie que coinciden con células mesenquimales de activación aberrante, las cuales podrían ser las progenitoras del tumor desmoide ${ }^{8}$.

La enfermedad presenta un curso silente de crecimiento lento e indoloro, con síntomas asociados a la infiltración o compromiso de los órganos adyacentes. Los casos de multifocalidad se evidencian con mayor frecuencia en las extremidades o por recurrencias posoperatorias ${ }^{8}$. Nuestra paciente debutó con un cuadro insidioso de dolor abdominal secundario a compresión gástrica. El estudio imagenológico de elección es la tomografía de abdomen contrastada, la cual permite una adecuada evaluación de las relaciones del tumor con los órganos intraabdominales y evidencia una lesión isodensa al músculo esquelético con densidad heterogénea en su interior que corresponde a colágeno $o$ áreas mixoides. En los casos de tumor desmoide en las extremidades, la evaluación con resonancia magnética es la indicada, con un mayor rendimiento respecto a la resonancia abdominal ${ }^{8}$.
El tumor desmoide está compuesto por células fusiformes parecidas a fibroblastos sin características de malignidad. Estas células se encuentran rodeadas por una matriz densa de colágeno y neovascularización de diversos calibres. Presentan inmunohistoquímica positiva para $\beta$-catenina con mayor expresión nuclear y vimentina. La expresión nuclear de la $\beta$-catenina no es específica para el tumor desmoide, pues puede estar presente en otras neoplasias mesenquimatosas, como el sarcoma sinovial, entre otras. El análisis de la mutación de CTNNB1 en los casos esporádicos es una herramienta diagnóstica en situaciones de poca claridad ${ }^{8}$.

El método de referencia sigue siendo la resección quirúrgica de la lesión. Actualmente es tema de debate la necesidad de garantizar unos márgenes microscópicos negativos, debido a que se reportan series de casos con tasas de recurrencia similares en grupos R0 y R1, por lo que se evalúa la administración de terapias complementarias con objetivo adyuvante. Hay que tener en cuenta, según la localización del tumor, la necesidad de resecciones multiviscerales y la morbilidad asociada al procedimiento; nuestro caso requirió, en su segunda intervención, la reconstrucción de la pared con material protésico separador de componentes ante el gran defecto posterior a la resección del tumor 8 .

Por otro lado, en cuanto al manejo con terapias sistémicas está indicado el uso de antiinflamatorios no esteroideos, como el sulindaco, y de hormonoterapia con efecto antiestrogénico, como el tamoxifeno o el raloxifeno ${ }^{9}$. Las terapias sistémicas como tratamiento de primera línea en los tumores intraabdominales asociados a poliposis adenomatosa familiar muestran una efectividad del $77 \%$, en comparación con pacientes a los se administró el tratamiento posterior a la aparición de recurrencia con una efectividad del 50\%.Otras terapias sistémicas son los agentes quimioterapéuticos como las antraciclinas, el metotrexato, la vinblastina y el cisplatino, con tasas de efectividad hasta del $79 \%$. Terapias también documentadas son los inhibidores de la tirosina cinasa y los antiangiogénicos que están en estudios de fase II mostrando resultados favorables ${ }^{10}$.

Finalmente, la radioterapia como adyuvante a la cirugía en casos de tumor desmoide cuando persisten márgenes microscópicamente positivos (R1) está indicada como terapia única ante una irresecabilidad quirúrgica de la lesión, obteniendo control local y estabilidad en crecimiento del $70-80 \%{ }^{4}$. De acuerdo con ciertas series de estudios, es posible lograr un 
control local del $78 \%$ con radioterapia sola y del $75 \%$ con radioterapia más cirugía, en comparación con el $61 \%$ con cirugía sola ${ }^{11}$. Para tumores desmoides intraabdominales es controversial el uso de la radioterapia por sus efectos adversos abdominales, como la enteritis actínica ${ }^{12}$.

\section{Conclusión}

La sospecha y la identificación de los tumores desmoides intraabdominales permiten realizar un manejo oportuno y preciso de una patología que, aunque poco frecuente, tiene un comportamiento localmente agresivo. En nuestro caso se logró una resección R1 (microscópicamente positivo), aceptable para dar un manejo adyuvante, aunque pendiente de estudios de mayor validación. Además, en esta paciente se descartó asociación con embarazo, trauma abdominal o cirugía, síndromes genéticos como el síndrome de Gardner y poliposis adenomatosa familiar.

\section{Agradecimientos}

A los pacientes del Instituto de Cancerología Las Américas, que permiten mediante el adecuado cumplimiento de buenas practicas clínicas la generación de evidencia científica.

\section{Conflicto de intereses}

Los autores declaran no tener ningún conflicto de intereses.

\section{Responsabilidades éticas}

Protección de personas y animales. Los autores declaran que para esta investigación no se han realizado experimentos en seres humanos ni en animales.

Confidencialidad de los datos. Los autores declaran que han seguido los protocolos de su centro de trabajo sobre la publicación de datos de pacientes.

Derecho a la privacidad y consentimiento informado. Los autores han obtenido el consentimiento informado de los pacientes y/o sujetos referidos en el artículo. Este documento obra en poder del autor de correspondencia.

\section{Bibliografía}

1. Williams AD, Heightchew K, Siripirapu V. Diagnostic and therapeutic dilemmas in intra-abdominal desmoid tumors: a case report and literature review. Int J Surg Case Rep. 2016;26:150-3.

2. Li W, Zhou Y, Li Q, Tong H, Lu W. Intestinal perforation during chemotherapeutic treatment of intra-abdominal desmoid tumor in patients with Gardner's syndrome: report of two cases. World J Surg Oncol. 2016;14:178.

3. Howard JH, Pollock RE. Intra-abdominal and abdominal wall desmoid fibromatosis. Oncol Ther. 2016;4:57-72.

4. Nuyttens JJ, Rust PF, Thomas CR Jr, Turrisi AT $3^{\text {rd }}$. Surgery versus radiation therapy for patients with aggressive fibromatosis or desmoid tumors: a comparative review of 22 articles. Cancer. 2000;88:1517-23.

5. Lips DJ, Barker N, Clevers H, Hennipman A. The role of APC and beta-catenin in the aetiology of aggressive fibromatosis (desmoid tumors). Eur J Surg Oncol. 2009;35:3-10.

6. Huss S, Nehles J, Binot E, Wardelmann E, Mittler J, Kleine MA, et al. $\beta$-catenin (CTNNB1) mutations and clinicopathological features of mesenteric desmoid-type fibromatosis. Histopathology. 2013;62:294-304.

7. Peng PD, Hyder O, Mavros MN, Turley R, Groeschl R, Firoozmand A, et al. Management and recurrence patterns of desmoids tumors: amulti-institutional analysis of 211 patients. Ann Surg Oncol. 2012; 19:4036-42.

8. Huang K, Wang CM, Chen JG, Du CY, Zhou Y, Shi YQ, et al. Prognostic factors influencing event-free survival and treatments in desmoid-type fibromatosis: analysis from a large institution. Am J Surg. 2014; 207:847-54

9. Gega M, Yanagi H, Yoshikawa R, Noda M, Ikeuchi H, Tsukamoto K, et al. Successful chemotherapeutic modality of doxorubicin plus dacarbazine for the treatment of desmoid tumors in association with familial adenomatous polyposis. J Clin Oncol. 2006;24:102-5.

10. De Camargo VP, Keohan ML, D'Adamo DR, Antonescu CR, Brennan MF, Singer $S$, et al. Clinical outcomes of systemic therapy for patients with deep fibromatosis (desmoid tumor). Cancer. 2010;116:2258-65.

11. Desurmont T, Lefevre JH, Shields C, Colas C, Tiret E, Parc Y. Desmoid tumour in familial adenomatous polyposis patients: responses to treatments. Fam Cancer. 2015;14:31-9.

12. Patel SR, Evans HL, Benjamin RS. Combination chemotherapy in adult desmoid tumors. Cancer. 1993;72:3244-7. 